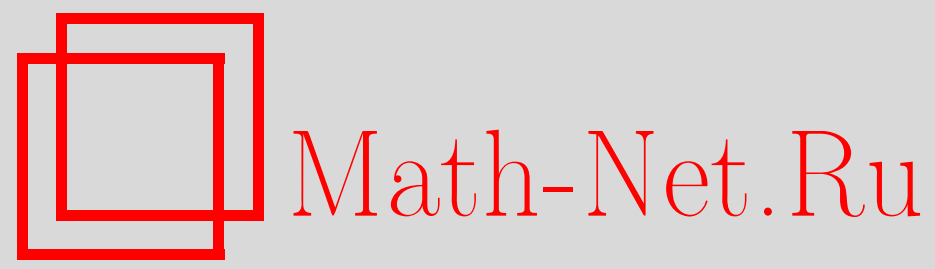

А. М. Зубков, Эйлер и комбинаторика, Совр. пробл. матем., 2008, выпуск 11, 5-18

DOI: https://doi.org/10.4213/spm20

Использование Общероссийского математического портала MathNet.Ru подразумевает, что вы прочитали и согласны с пользовательским соглашением

http://www . mathnet.ru/rus/agreement

Параметры загрузки:

IP : 54.224 .60 .19

26 апреля 2023 г., 16:44:37

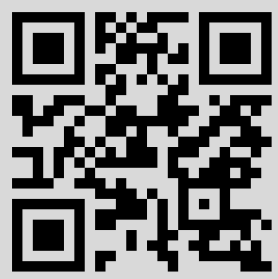




\title{
Эйлер и комбинаторика
}

\author{
А. М. Зубков
}

Леонард Эйлер является одним из создателей методов математического анализа. Наряду со строгими математическими (дедуктивными) доказательствами Эйлер широко использовал эмпирические методы (индукцию) и "правдоподобные" рассуждения. Развитие методов математического анализа в значительной степени проводилось Эйлером с целью решения конкретных математических и прикладных задач. Работы Эйлера внесли большой вклад в разные области математики, в том числе и в комбинаторику. Комбинаторные результаты Леонарда Эйлера, лежащие несколько в стороне от основной массы его работ, позволяют лучше оценить как разносторонность его дарования, так и склонность к использованию аналитических методов.

Термин "комбинаторная математика" разные авторы понимают по-разному. Начиная с древних греков, главной математической абстракцией является бесконечность (в виде бесконечного ряда натуральных чисел, безграничной делимости отрезка прямой, неограниченности пространства и т. п.). Немного утрируя, можно сказать, что в отличие от почти всех остальных областей математики, которые в том или ином виде связаны с изучением свойств бесконечных объектов (неограниченных или состоящих из бесконечного множества элементов), комбинаторика включает в себя задачи, относящиеся к свойствам конечных (во всех смыслах) объектов.

Ниже приводится краткий обзор исследований Эйлера по направлениям, которые называют теперь теорией графов, теорией латинских квадратов, перечислительной комбинаторикой. Обсуждаются методы, использовавшиеся Эйлером, и некоторые более поздние результаты в этих направлениях, связанные с работами Эйлера.

(c) Зубков А. М., 2008 


\section{1. Теория графов}

Одной из наиболее известных комбинаторных работ Леонарда Эйлера является статья с решением задачи о мостах города Кёнигсберг [8].

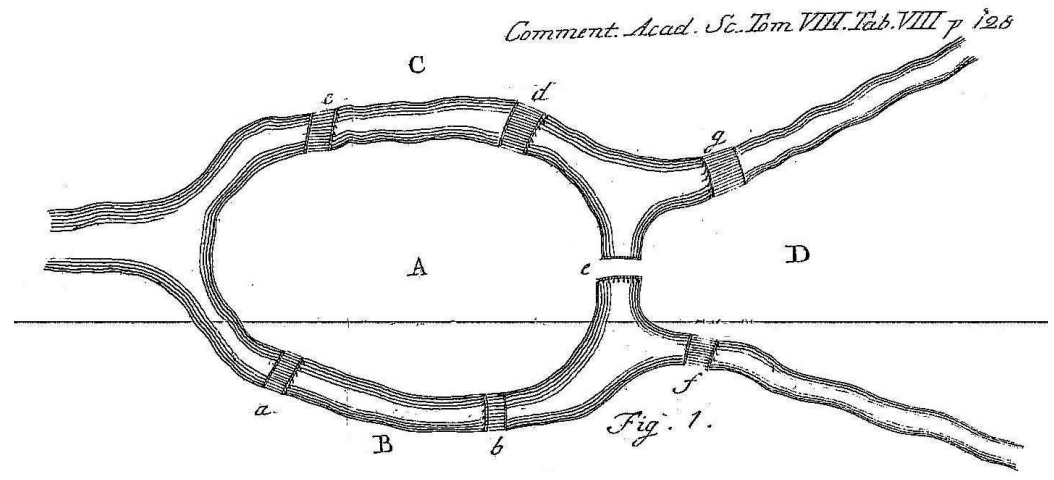

Рис. 1. Схема мостов Кёнигсберга из статьи Эйлера [8].

Река, протекающая через Кёнигсберг, делит его на 4 части, которые были соединены 7 мостами (рис. 1). Существовало поверье, согласно которому загаданное желание должно исполниться, если пройти по каждому из 7 мостов ровно по одному разу. Лейбниц в переписке с Вольфом в 1670 г. обсуждал математическую задачу о существовании маршрута обхода всех кёнигсбергских мостов и высказывал мнение о том, что она представляет собой новый тип геометрических задач, связанных не с размерами объектов, а только с их взаимным расположением (теперь такие задачи называются топологическими), и что поэтому для ее решения нужно использовать качественно новые методы.

Эйлер в своей работе [8] предложил строгое, изящное и в конечном счете аналитическое решение, основанное на правильной переформулировке задачи о мостах. Рассмотрим идеи этого решения.

Прием, использованный Эйлером, теперь иногда называют “двойным счетом" (одна и та же величина вычисляется двумя 
разными способами). Прежде всего, в качестве основных объектов Эйлер использует не мосты, а соединяемые ими области суши. Если обозначать области суши прописными латинскими буквами, то маршрут прохода по мостам можно представить последовательностью $A B C A D \ldots$ символов областей. (Такая запись не отражает, в каком порядке проходит маршрут по мостам, соединяющим две области, если таких мостов несколько, но оказывается, что это и не существенно!) Далее, число символов в маршруте, который проходит по каждому мосту один раз, на 1 больше числа мостов в маршруте, поскольку каждая пара соседних символов соответствует переходу по одному мосту. Наконец, основное соображение состоит в том, что если символ области $X$ не является концевой точкой маршрута, то он соответствует проходу по двум мостам, соединяющим область $X$ с другими областями, а если символ $X$ является концевой точкой - то проходу по одному такому мосту. Отсюда следует, что если маршрут проходит по $N$ мостам, соединяющим область $X$ с другими областями, то символ $X$ появляется в записи маршрута $(N+1) / 2$ раз, если $N$ нечетное (и при этом один раз $X$ является концевой точкой маршрута), и либо $N / 2$, либо $N / 2+1$ раз, если $N$ четное (в последнем случае маршрут начинается и заканчивается символом $X)$.

Правило “двойного счета" Эйлер попутно применяет еще раз, замечая, что у каждого моста два конца и что поэтому сумма чисел мостов, соединяющих каждую из областей с остальными, вдвое превосходит общее число мостов и поэтому должна быть четной; но тогда число областей, в которых заканчивается нечетное число мостов, тоже должно быть четным.

Из этих соображений Эйлер выводит, что если число мостов равно $M$, а число областей с нечетным числом мостов равно $2 m$, то запись маршрута, проходящего по всем мостам, должна содержать не менее $M+m$ символов, что больше $M+1$, если $m>1$. Это условие позволяет убедиться, что не существует маршрута, проходящего по 7 мостам Кёнигсберга по одному разу. Эйлер не описывает подробно способ построения маршрута в ситуациях, когда $m \leqslant 1$, ограничиваясь замечаниями о том, что при $m=1$ маршрут должен начинаться и заканчиваться в областях с нечетным числом мостов и что если две области соединены несколькими мостами, то можно мысленно удалить пары таких мостов и получить более простую схему, а после того, как обход упрощен- 
ной схемы будет построен, можно добавить к нему проходы по удаленным мостам.

Интересно отметить, как Эйлер относился к своему решению. В [10] среди других писем Эйлера приводится письмо Карлу Леонарду Готлибу Элеру (Carl Leonard Gottlieb Ehler), любителю математики и бургомистру г. Данцига, в котором Эйлер по просьбе Элера кратко излагает свое решение задачи о мостах, а затем пишет:

"Следовательно, тъ можешь убедитъся, славнейший муж, что это решение по своему характеру имеет мало отношения $\kappa$ математике, и мне непонятно, почему следует скорее от математика ожидать этого решения, нежели от какого-нибудь другого человека, ибо это решение подкрепляется одним только рассуждением, и нет необходимости привлекать для нахождения этого решения какие-либо законы, свойственные математике. Итак, я не знаю, каким образом получается, что вопросы, имеюшие совсем мало отношения $\kappa$ математике, скорее разрешаются математиками, чем другими [ученъми]".

По-видимому, Эйлер понимал математику главным образом как науку о вычислениях; но он живо интересовался также качественно новыми задачами, как показывает продолжение приведенного отрывка:

"Между тем Ты, славнейший муж, определяешь место этого вопроса в геометрии положения, и что касается этой новой науки, то, признаюсь, мне неизвестно, какого рода относящиеся сюда задачи желательны были Лейбницу и Вольфу. Итак, я прошу Тебя, если Ть считаешь, что я способен нечто создать в этой новой науке, чтобы Ты соблаговолил мне прислать несколько определенных, относящихся $\kappa$ ней задач, для того, чтобы я мог лучше уяснить себе, что именно представляется желательным. Между тем, поскольку мне известно, что Ты также можешь составить суждение о математических науках на основании одной только задачи, которую следует предложить u решить, начало чему по Твоей просъбе положил преславный Кюн, я хотел бы, чтобы он представил нам такого рода задачи, которые он считает более трудными, [сделав это] как для прогресса науки, так и для упражнения наших сил."

В современной теории графов путь, проходящий по одному разу по всем ребрам графа, называют эйлеровым путем, а за- 
мкнутый эйлеров путь - эйлеровым ииклом. Найденный Эйлером критерий в современных терминах выглядит так:

"В связном неориентированном графе эйлеров путь (цикл) существует тогда и только тогда, когда число вершин нечетной кратности не больше 2 (вершин нечетной кратности нет)".

Более общий критерий охватывает случай, когда в графе есть как ориентированные, так и неориентированные ребра:

"В связном графе эйлеров путь (цикл) существует тогда и только тогда, когда в графе с удаленными ориентациями ребер существует эйлеров путь (цикл) и для каждой вершины разность между числом входящих и выходящих ребер не превышает числа инцидентных этой вершине неориентированных ребер."

После решения задачи о мостах Эйлер, видимо, продолжал размышлять о "геометрии положений", и в 1750 г. в письме Гольдбаху (см. также [4]) он сообщил о найденной им формуле, связывающей число $V$ вершин, число $E$ ребер и число $F$ граней выпуклого многогранника: $N-E+F=2$. Первое строгое доказательство этой формулы, которую называют теперь формулой Эйлера, опубликовал О. Коши [3] в 1813 г., когда ему было около 20 лет. Доказательство Коши является индуктивным и использует наглядные, а не аналитические, соображения. В 1893 г. А. Пуанкаpe [15] обобщил формулу Эйлера на $n$-мерные политопы: если $N_{k}$ - число $k$-мерных $(k=0,1, \ldots, n-1)$ граней, то

$$
N_{0}-N_{1}+N_{2}-\cdots+(-1)^{n-1} N_{n-1}=1+(-1)^{n-1} .
$$

Обобщения выражения, стоящего в левой части, используются как топологические инварианты поверхностей.

В “задаче о мостах" рассматриваются пути, проходящие по всем ребрам графа. В связи с ней естественно возникает аналогичная задача о путях, проходящих по одному разу через все вершины графа; такие пути называются гамильтоновыми. В общем случае задача выяснения вопроса о существовании гамильтонова пути в графе является NP-полной, т. е. принадлежит множеству $\Sigma$ таких бесконечных совокупностей $\sigma$ задач, что: а) ни для какой совокупности $\sigma \in \Sigma$ не известен алгоритм, решающий любую задачу из $\sigma$ за время, полиномиально зависящее от длины формулировки задачи, и б) если для какой-нибудь совокупности $\sigma \in \Sigma$ существует алгоритм, решающий любую задачу из $\sigma$ за полиномиальное время, то для любой другой совокупности $\sigma^{\prime} \in \Sigma$ тоже 
существует алгоритм, решающий все задачи из $\sigma^{\prime}$ за полиномиальное время.

Таким образом, в ближайшей окрестности решенной Эйлером "задачи о мостах" находятся задачи, не решенные до сих пор.

В работе [9], представленной к публикации в 1759 г. и опубликованной в 1766 г., Леонард Эйлер рассмотрел задачи построения обходов шахматной доски (и ее обобщений) шахматным конем, которые являются частными случаями задачи о гамильтоновых путях. Это была первая серьезная математическая статья, посвященная способам построения обходов квадратных и прямоугольных досок ходом шахматного коня; она определила характер дальнейших исследований в этой области "занимательной математики". Эйлер построил замкнутый обход шахматной доски (доказав тем самым, что обход можно начинать с любой клетки) и указал способы преобразования и построения обходов и привел разнообразные примеры обходов (как замкнутых, так и не замкнутых, в том числе обладающих различными свойствами симметрии). Кроме того, Эйлер рассмотрел задачи об обходе конем досок, имеющих другие размеры и формы. В частности, он:

а) отметил, что не существует обходов досок размеров $3 \times 3$ и $4 \times 4$ и привел пример обхода конем всех клеток доски $4 \times 4$, кроме одной угловой клетки;

б) заметил, что не существует замкнутых обходов досок с нечетным числом клеток, показал, что любой обход доски размером $5 \times 5$ должен начинаться или заканчиваться в угловой клетке, и, соединив обходы досок $5 \times 5$, построил симметричный обход доски $10 \times 10$;

в) построил (незамкнутые) обходы досок размером $3 \times 4$ и $3 \times 7$, отметил, что доски размером $3 \times 5$ и $3 \times 6$ обойти ходом шахматного коня нельзя, и высказал гипотезу о том, что не существует замкнутых обходов досок, у которых хотя бы одна из сторон короче 5 клеток;

г) построил примеры обходов досок крестообразной формы.

Гипотеза Эйлера была опровергнута только в 1917 г., когда Е. Бергхольт в письмах в The British Chess Magazine привел примеры замкнутых обходов досок размером $3 \times 10$ и $3 \times 12$. 


\section{2. Магические и латинские квадраты}

Две работы Леонарда Эйлера, посвященные магическим и латинским квадратам, заложили основы нового направления комбинаторики - теории латинских квадратов, результаты которой применяются в различных областях.

Таблица размера $n \times n$, заполненная натуральными числами от 1 до $n^{2}$, называется магическим квадратом, если суммы чисел во всех ее строках, столбцах и в двух диагоналях одинаковы. Магические квадраты были известны еще в древнем Китае, и считалось, что они могут обладать волшебными свойствами.

Таблица размера $n \times n$, заполненная натуральными числами от 1 до $n$, называется латинским квадратом, если в каждой строке и в каждом столбце находится перестановка чисел от 1 до $n$.

Первое упоминание о латинских квадратах (в связи с решением карточных задач) относится к 1723 г. Систематическое изучение латинских квадратов началось с работ Эйлера [6], [7].

В [6] Эйлер предложил для построения магических квадратов использовать представление целых чисел от 0 до $n^{2}-1$ в виде сумм $a n+b$, где $a$ и $b$ принимают значения от 0 до $n-1$. Если $\left\|a_{i j}\right\|_{i, j=1}^{n}$ и $\left\|b_{i j}\right\|_{i, j=1}^{n}$ - два латинских квадрата, заполненных числами от 0 до $n-1$, и все $n^{2}$ элементов квадратной таблицы $\left\|\left(a_{i j}, b_{i j}\right)\right\|$ различны, то в квадратной таблице $\left\|a_{i j} n+b_{i j}\right\|$ суммы чисел, стоящих в любой строке и в любом столбце, равны одному и тому же числу $\frac{1}{2} n(n-1) n+\frac{1}{2} n(n-1)$. (Эйлер использовал для обозначения чисел $a_{i j}$ латинские, а для чисел $b_{i j}$ - греческие буквы и поэтому назвал таблицу $\left\|\left(a_{i j}, b_{i j}\right)\right\|$ греко-латинским квадратом; этот термин иногда используется и в наше время, но чаще латинские квадраты $\left\|a_{i j}\right\|_{i, j=1}^{n}$ и $\left\|b_{i j}\right\|_{i, j=1}^{n}$, для которых все пары $\left(a_{i j}, b_{i j}\right)$ различны, называют ортогональными.)

На примерах квадратов размеров $3 \times 3,4 \times 4,5 \times 5$ Эйлер показал, что условие равенства диагональных сумм суммам по строкам и столбцам при такой конструкции записывается в виде линейных уравнений, выполнение которых можно обеспечить за счет переобозначения элементов. Однако построить греколатинский квадрат размера $6 \times 6$ Эйлеру не удалось, и он построил магический квадрат размера $6 \times 6$, комбинируя квадраты, не являющиеся латинскими.

По-видимому, неудача с реализацией внешне простого способа построения магического квадрата размера $6 \times 6$ с помощью двух 
греко-латинских (ортогональных) латинских квадратов, заинтересовала Эйлера, и он посвятил большую работу [7] изучению способов построения латинских и греко-латинских квадратов. В частности, в ней были указаны способы построения ортогональных латинских квадратов размера $n \times n$ при $n \not \equiv 2(\bmod 4)$, и Эйлер высказал предположение о том, что при $n \equiv 2(\bmod 4)$ ортогональных латинских квадратов размера $n \times n$ не существует. В случае $n=6$ Эйлер сформулировал свою гипотезу в виде задачи о том, можно ли расставить 36 офицеров из 6 полков (по 6 разных чинов из каждого полка) в виде квадрата $6 \times 6$ так, чтобы в каждом столбце и в каждой строке находились офицеры разных званий и из разных полков. Эту гипотезу Эйлера частично подтвердил в 1900 г. G. Tarry [16], доказав (фактически - перебором всех возможных неэквивалентных пар латинских квадратов размера $6 \times 6$ ), что в случае $n=6$ она верна. Следующее продвижение в исследовании гипотезы Эйлера было сделано лишь в 1959 г., когда Bose, Shrikhande и Parker [2], [14], [1] установили, что пары ортогональных латинских квадратов существуют при всех натуральных $n \neq 2,6$.

Таким образом, для того, чтобы исправить утверждение сформулированной Эйлером гипотезы, потребовалось почти 200 лет развития как математики, так и техники (примеры ортогональных латинских квадратов порядка 10 были построены с помощью ЭВМ). Можно сказать, что трудоемкость задач, не поддававшихся гению Эйлера, превышает 100 лет коллективной работы других математиков.

Одна из пар ортогональных латинских квадратов порядка 10 изображена на рис. 2.

Для различных значений $n$ существует разное число попарно ортогональных латинских квадратов. Число попарно ортогональных латинских квадратов порядка $n$ не может быть больше $n-1$, и это значение достигается в случаях, когда $n-$ степень простого числа (такая система попарно ортогональных латинских квадратов называется полной). Общей формулы для максимального числа попарно ортогональных латинских квадратов порядка $n$ не существует; более того, до сих пор неизвестно, существуют ли три попарно ортогональных квадрата порядка 10.

В XVIII веке, когда Эйлер ввел понятие греко-латинских (opтогональных) квадратов, они были просто новыми чисто математическими объектами. В дальнейшем латинские и особенно орто- 


\begin{tabular}{|l|l|l|l|l|l|l|l|l|l|}
\hline 11 & 87 & 96 & 05 & 20 & 49 & 68 & 32 & 53 & 74 \\
\hline 78 & 22 & 81 & 97 & 06 & 30 & 59 & 43 & 64 & 15 \\
\hline 69 & 18 & 33 & 82 & 91 & 07 & 40 & 54 & 75 & 26 \\
\hline 50 & 79 & 28 & 44 & 83 & 92 & 01 & 65 & 16 & 37 \\
\hline 02 & 60 & 19 & 38 & 55 & 84 & 93 & 76 & 27 & 41 \\
\hline 94 & 03 & 70 & 29 & 48 & 66 & 85 & 17 & 31 & 52 \\
\hline 86 & 95 & 04 & 10 & 39 & 58 & 77 & 21 & 42 & 63 \\
\hline 23 & 34 & 45 & 56 & 67 & 71 & 12 & 88 & 90 & 09 \\
\hline 35 & 46 & 57 & 61 & 72 & 13 & 24 & 00 & 89 & 98 \\
\hline 47 & 51 & 62 & 73 & 14 & 25 & 36 & 99 & 08 & 80 \\
\hline
\end{tabular}

Рис. 2. Ортогональные латинские квадраты порядка 10.

гональные латинские квадраты нашли применения в различных областях (см., например, [13]).

В комбинаторике полные системы ортогональных латинских квадратов соответствуют конечным аффинным и проективным плоскостям. Латинские квадраты используются при построении квадратов Рума (турниров игры в бридж). В конце XIX века Кэли показал, что таблица умножения элементов конечной группы является латинским квадратом. В 30-х годах XX века возникло понятие квазигруппы, в которой таблицей умножения может быть любой латинский квадрат.

Системы попарно ортогональных латинских квадратов используются при построении сеточных методов интегрирования в вычислительной математике.

В 30-х годах ХХ века Р. Фишер [11], [12] предложил использовать латинские (и ортогональные латинские) квадраты для планирования сельскохозяйственных экспериментов. Число таких экспериментов не может быть произвольно большим (хотя бы потому, что каждый эксперимент длится несколько месяцев), на результат эксперимента могут оказывать влияние несколько факторов, в том числе и неконтролируемые. Например, пусть в математической модели результат эксперимента линейно зависит от трех контролируемых факторов, каждый из которых принимает $n$ разных значений, и от случайной величины. Обозначим возможные влияния каждого из факторов числами $\alpha_{1}, \ldots, \alpha_{n}$, $\beta_{1}, \ldots, \beta_{n}, \gamma_{1}, \ldots, \gamma_{n}$; тогда результат эксперимента, в котором факторы принимают соответственно $i$-е, $j$-е и $k$-е значения, ра- 
вен

$$
x_{i j k}=\mu+\alpha_{i}+\beta_{j}+\gamma_{k}+\varepsilon_{i j k},
$$

где $\mu$ - среднее значение результата, а $\varepsilon_{i j k}$ - случайное влияние. Чтобы проверить справедливость статистической гипотезы о том, что $\alpha_{i}=\beta_{i}=\gamma_{i}=0, i=1, \ldots, n$, т. е. что ни один фактор не влияет на результат эксперимента, можно поставить $n^{3}$ экспериментов со всеми возможными сочетаниями факторов. Однако число экспериментов можно сократить до $n^{2}$, если использовать латинский квадрат $\left\|a_{i j}\right\|_{i, j=1}^{n}$ и провести $n^{2}$ экспериментов со следующими сочетаниями факторов: $\left(i, j, a_{i j}\right), i, j=1, \ldots, n$.

Еще одна область применения латинских квадратов - построение кодов, исправляющих ошибки. Кодом $C$ длины $N$ над алфавитом $A$ из $n$ букв называется множество $N$-мерных векторов (кодовых слов), компоненты которых принадлежат A. Расстоянием $d(C)$ кода $C$ называется минимальное расстояние Хемминга (число несовпадающих координат) между элементами кода. При передаче кодовых слов могут происходить искажения отдельных координат. Если число искаженных координат меньше $\frac{1}{2} d(C)$, то по искаженному кодовому слову можно однозначно восстановить неискаженное. Поэтому говорят, что код $C$ исправляет $\left[\frac{1}{2} d(C)\right]$ ошибок. Если число искаженных координат меньше $d(C)$, то однозначное восстановление кодового слова может оказаться невозможным, но факт наличия искажений обнаруживается.

Ортогональные латинские квадраты позволяют строить коды, исправляющие ошибки. Пусть $A_{1}=\left\|a_{i j}^{(1)}\right\|, \ldots, A_{m}=\left\|a_{i j}^{(m)}\right\|$ - попарно ортогональные латинские квадраты порядка $n$. Тогда множество кодовых слов длины $m+2$

$$
\left(i, j, a_{i j}^{(1)}, \ldots, a_{i j}^{(m)}\right), \quad i, j=1, \ldots, n,
$$

образует код из $n^{2}$ элементов, исправляющий $[m / 2]$ ошибок. Действительно, достаточно показать, что любые два кодовых слова различаются по крайней мере $m+1$ координатой. Любые два разных кодовых слова $\left(i, j, a_{i j}^{(1)}, \ldots, a_{i j}^{(m)}\right),\left(i^{\prime}, j^{\prime}, a_{i^{\prime} j^{\prime}}^{(1)}, \ldots, a_{i^{\prime} j^{\prime}}^{(m)}\right)$ различаются хотя бы одной из первых двух координат. Если, например, $i=i^{\prime}$, то $j \neq j^{\prime}$ и $a_{i j}^{(k)} \neq a_{i j^{\prime}}^{(k)}, k=1, \ldots, m$, так как в каждом латинском квадрате все числа в $i$-й строке попарно различны. Аналогично рассматривается случай $i \neq i^{\prime}, j=j^{\prime}$. Если же $i \neq i^{\prime}, j \neq j^{\prime}$, то может существовать не более одного такого $k$, что $a_{i j}^{(k)}=a_{i^{\prime} j^{\prime}}^{(k)}$, так как если $a_{i j}^{\left(k_{1}\right)}=a_{i^{\prime} j^{\prime}}^{\left(k_{1}\right)}, a_{i j}^{\left(k_{2}\right)}=a_{i^{\prime} j^{\prime}}^{\left(k_{2}\right)}$, то 


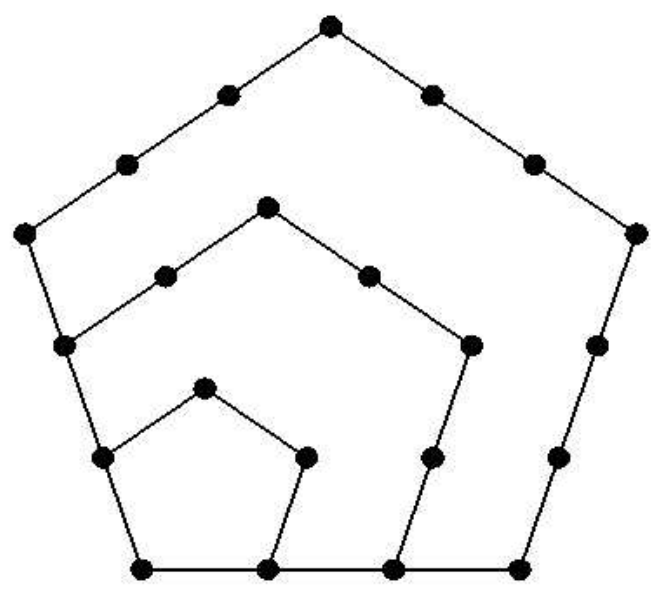

Рис. 3. Пятиугольные числа: $1,5,12,22, \ldots$.

$\left(a_{i j}^{\left(k_{1}\right)}, a_{i j}^{\left(k_{2}\right)}\right)=\left(a_{i^{\prime} j^{\prime}}^{\left(k_{1}\right)}, a_{i^{\prime} j^{\prime}}^{\left(k_{2}\right)}\right)$, т. е. латинские квадраты $A_{k_{1}}$ и $A_{k_{2}}$ не ортогональны.

Вряд ли Эйлер предполагал, что латинские квадраты будут столь широко применяться, однако его математическая интуиция помогла правильно оценить естественность конструкции и нетривиальность свойств латинских квадратов.

\section{3. Теорема о пятиугольных числах}

Наконец, необходимо упомянуть еще один характерный для Леонарда Эйлера цикл исследований, находящийся на стыке комбинаторики, теории чисел и математического анализа и посвященный свойствам пятиугольных чисел. Так называемые многоугольные числа определяются как элементы последовательностей натуральных чисел, образованных количествами "целых" точек, содержащихся в гомотетичных многоугольниках с целочисленными длинами сторон (рис. 3).

Многоугольные числа были известны еще в древней Греции. $\mathrm{B}$ частности, формула $n$-го пятиугольного числа имеет вид $p_{n}=$ $\frac{1}{2} n(3 n-1), n=1,2, \ldots$ Судя по переписке, около 1740 г. Эйлер обнаружил, что при разложении бесконечного произведения

$$
(1-x)\left(1-x^{2}\right)\left(1-x^{3}\right)\left(1-x^{4}\right) \cdots
$$


в степенной ряд получается выражение

$$
1-x-x^{2}+x^{5}+x^{7}-x^{12}-x^{15}+x^{22}+x^{26}-x^{35}-x^{40}+x^{51}+\cdots,
$$

в котором присутствуют не все степени $x$ и в котором ненулевые коэффициенты равны \pm 1 . Эйлер заметил, что все показатели степеней с ненулевыми коэффициентами имеют вид $\frac{1}{2} n(3 n \pm 1)$; это множество обобщенных пятиугольных чисел содержит как обычные пятиугольные числа $p_{1}, p_{2}, \ldots$, так и числа, которые получаются при подстановке в формулу $\frac{1}{2} n(3 n-1)$ значений $n=0,-1,-2, \ldots$ Строгое доказательство теоремы о пятиугольных числах, т. е. тождества

$$
\prod_{n=1}^{\infty}\left(1-x^{n}\right)=1+\sum_{n=1}^{\infty}(-1)^{n}\left(x^{\frac{n(3 n-1)}{2}}+x^{\frac{n(3 n+1)}{2}}\right)
$$

Эйлер нашел только в 1750 г., а опубликовал в 1760 г. [5]. Эта удивительная теорема стала первым примером нетривиальных тождеств, связывающих бесконечные произведения и степенные ряды; ряд других неожиданных тождеств был найден в начале XX века индийским математиком С. Рамануджаном.

Эйлер показал также, что другая эмпирически обнаруженная им в 1747 г. серия необычных равенств, связанных с пятиугольными числами, является следствием теоремы о пятиугольных числах. Пусть $\sigma(n)$ - сумма всех натуральных делителей числа $n$. Эйлер показал, что при любом $n$

$$
\begin{gathered}
\sigma(n)=\sigma(n-1)+\sigma(n-2)-\sigma(n-5)-\sigma(n-7)+ \\
+\sigma(n-12)+\sigma(n-15)-\cdots ;
\end{gathered}
$$

при каждом $n$ в сумму в правой части входят только те слагаемые, аргументы которых положительны. Нетрудно заметить, что структура суммы в правой части (2) совпадает со структурой ряда (1).

\section{Заключение}

Подводя итог этому обзору, можно отметить, что, во-первых, Леонард Эйлер получил первые важные результаты в нескольких направлениях комбинаторики и сформулировал в качестве гипотез задачи, ставшие ориентирами для развития этих направлений 
более чем на 100 последующих лет; во-вторых, основной заслугой Эйлера перед комбинаторикой следует считать не столько введение новых понятий и решение конкретных задач, сколько разработку методов использования степенных рядов и производящих функций для получения точных и асимптотических формул в перечислительных задачах.

\section{Список литературы}

[1] R. C. Bose, S. S. Shrikhande, E. T. Parker, "Further results on the construction of mutually orthogonal Latin squares and the falsity of Euler's conjecture", Canad. J. Math., 12 (1960), 189-203 MR 122729, Zbl 0093.31905.

[2] R. C. Bose, S. S. Shrikhande, "On the falsity of Euler's conjecture about the nonexistence of two orthogonal Latin squares of order $4 t+2 "$, Proc. Nat. Acad. Sci. USA, 45 (1959), 734-737 doi 10.1073/pnas.45.5.734, MR 0104590, Zbl 0085.00902, ADS 1959PNAS . . .45 . .734B.

[3] A. Cauchy, "Recherches sur les polyèdres", J. École Polytechnique, XVI Cahier, IX (1813), 68-83.

[4] L. Euler, "Elementa doctrinae solidorum - Demonstratio nonnullarum insignium proprietatum, quibus solida hedris planis inclusa sunt praedita", Novi commentarii Academiae scientiarum imperialis Petropolitanae, 4 (1752-3), 109-140.

[5] L. Euler, "Demonstratio theorematis circa ordinem in summis divisorum observatum", Novi commentarii Academiae scientiarum imperialis Petropolitanae, 5 (1760), 75-83.

[6] L. Euler, "De quadratis magicis", Представлено СанктПетербургской Академии Наук 17 октября 1776 г., впервые опубликовано в Mem. Soc. Flessingue, Comm. Arith. Collect. (eloge St.Petersburg 1783) 2 (1849), 593-602.

[7] L. Euler, "Recherches sur une nouvelle espece de quarres magiques", Представлено Санкт-Петербургской Академии Наук 8 марта 1779 г., впервые опубликовано в Verhandelingen uitgegeven door het Zeeuwsch Genootschap der Wetenschappen te Vlissengen 9 (1782), 85239; Commentationes arithmeticae, 1 (1849), 302-361; Opera Omnia, Ser. I, 7, 291-392.

[8] L. Euler, "Solutio Problematis ad Geometriam Situs Pertinentis", Commentarii Academiae Scientiarum Imperialis Petropolitanae, 8 (1741), 128-140; Opera Omnia (1), 7, 1-10.

[9] L. Euler, "Solution d'une question curieuse que ne paroit soumise ò aucune analyse", Mémoires de l'académie des sciences de Berlin, 
15 (1759) (1766), 310-337; Commentationes arithmeticae, 1 (1849), 337-355; Opera Omnia, Ser. I, 7, 26-56.

[10] Л. Эйлер, Писъма к ученым, изд-во АН СССР, М.-Л., 1963.

[11] R. A. Fisher, Statistical methods for research workers, Oliver \& Boyd, Edinburg, London, 1925.

[12] R. A. Fisher, "The arrangement of field experiments", J. Ministry of Agriculture, 33 (1926), 503-513.

[13] Ch. F. Laywine, G. L. Mullen, Discrete mathematics using Latin squares, J. Wiley \& Sons, New York, 1998 MR 1644242.

[14] E. T. Parker, "Orthogonal Latin squares", Proc. Nat. Acad. Sci. USA, 45 (1959), 859-862 doi 10.1073/pnas.45.6.859, MR 0104591, Zbl 0086.02201, ADS 1959PNAS . . 45 . 859P.

[15] H. Poincaré, "Analysis Situs", J. École Polytechnique 2, 1 (1895).

[16] G. Tarry, "Le problème des 36 officers", Compt. Rend. Ser. Math., Astron., Géod. Mécan., 29 (1900), 170-203. 